07

\title{
Исследование электронной прозрачности графена для малых энергий электрона
}

\author{
() Э.А. Ильичев, А.Е. Кулешов, Д.М. Мигунов, Р.М. Набиев, \\ Г.Н. Петрухин, Г.С. Рычков Ф, Е.Г. Теверовская, В.О. Хаустов
}

Национальный исследовательский университет „Московский институт электронной техники“, Зеленоград, Москва, Россия

『 E-mail: mstlena2@mail.ru

\section{Поступило в Редакцию 25 апреля 2018 г.}

Исследуется прозрачность графеновых мембран для электронов с энергией в диапазоне 5-50 eV. Предполагается использование графена как электрода, стимулирующего полевую эмиссию в вакуумных микро- и наноэлектронных устройствах. При анализе поведения отраженных от мембраны электронов учитывается их возврат под действием тормозящего электрического поля. В качестве электронов с небольшой энергией используются фотоэлектроны, эмитируемые из алмазного фотокатода под действием вакуумного ультрафиолета.

DOI: 10.21883/PJTF.2018.18.46617.17356

Благодаря своим уникальным электронным и механическим свойствам графен привлекает внимание многих разработчиков электронной аппаратуры. Особенно следует отметить уникальные свойства графеновых мембран как микро- и нанорезонаторов, обеспечивающих разработку автоколебательных систем для передачи и вывода информации из микро- и наносистем [1]. Генерация высокочастотных колебаний достигается введением положительной обратной связи, и в дальнейшем для этой цели предполагается использовать CMOSтехнологию. Однако необходимую связь можно реализовать, используя графеновые мембраны не только как резонаторы, но и как электроды, стимулирующие полевую эмиссию [2,3]. Тогда можно получить наноразмерный автогенератор, превосходящий по размерам автогенератор на основе туннельно-резонансных приборов [4]. Существенным при этом становится свойство электронной прозрачности графеновой мембраны, чему посвящен ряд работ [2,3,5-7], результаты которых довольно противоречивы. Это связано с исследованием образцов, по структуре сильно 
отличающихся друг от друга. В [8] выполнены квантово-механические расчеты для энергий 20-200 eV. Результаты, полученные для $20-40 \mathrm{eV}$, показывают резкие колебания прозрачности в зависимости от энергии, что, по мнению самих авторов указанной работы, является странным и, возможно, связано с недостаточной точностью в аппроксимации графена потенциальным полем. Кроме того, при расчетах полагалось, что электрон падает нормально к поверхности графена. В реальности это не так, но учет данного факта сильно усложняет расчеты, что обусловлено возникновением резонансных явлений $[9,10]$.

Использование графенового электрода в качестве управляющей сетки в вакуумных триодных структурах характеризуется тем, что отраженные от графена электроны попадают в поле, возвращающее их обратно на графен или фиксирующую его подложку. Это приводит к тому, что часть потока проходит через графен, а остальная часть поглощается непрозрачной подложкой, на которой располагаются графеновые мембраны. Следует также отметить работу [5], в которой в качестве источника электронов использовался напыленный термокатод $\mathrm{BaO}: \mathrm{W}$, нагреваемый до $800^{\circ} \mathrm{C}$. Показано, что при энергии электронов от 3 до $10 \mathrm{eV}$ наблюдался почти линейный рост прозрачности (от 0.1 до 0.6). При дальнейшем увеличении энергии коэффициент прозрачности колебался, но не увеличивался, что объяснялось возникновением пространственного заряда. С течением времени из-за испарения с катода Ва и его осаждения на графене прозрачность сильно падала. В работе поднимается ряд дополнительных вопросов и показана проблематичность использования термокатода.

Графеновые мембраны перспективно использовать для стимуляции полевой эмиссии $[2,3,11]$. В работе [11] показано, что в этом случае мембрана позволяет создать когерентный источник электронов для голографического исследования наноразмерных объектов, причем при энергии электронов $\sim 66 \mathrm{eV}$ прозрачность мембраны достигает 0.73 .

Приведенный обзор показывает, что изучение электронной прозрачности графена для электронов малой энергии следует продолжить. В настоящей работе экспериментально исследована прозрачность графена для энергий электрона $5-50 \mathrm{eV}$.

Схема экспериментальной установки приведена на рис. 1, a. Установка состоит из фотокатода 1 , представляющего собой сапфировую пластину, на поверхности которой сформирован слой поликристаллического алмаза р-типа проводимости, сеточного анода 2 и оснастки 3 ,

Письма в ЖТФ, 2018, том 44, вып. 18 

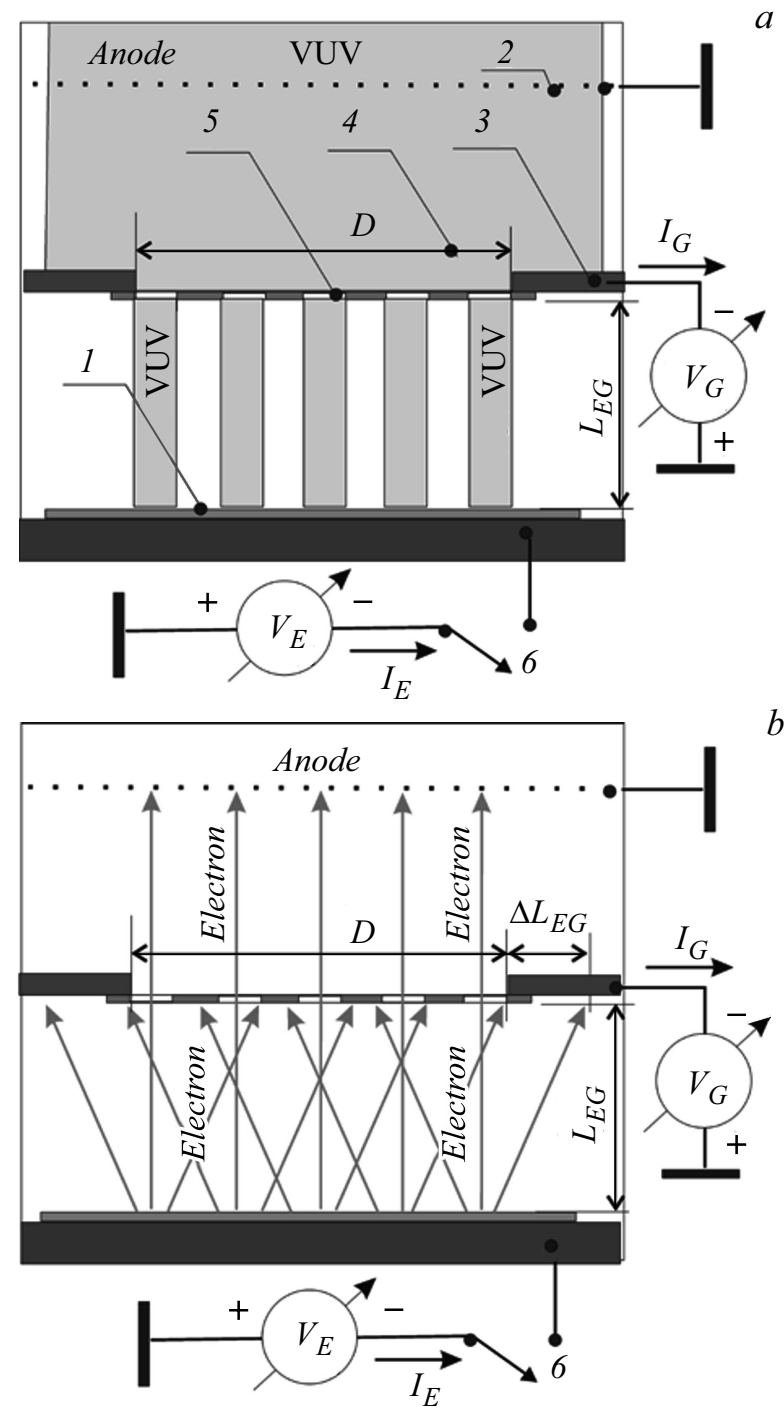

Pис. 1. Схема экспериментальной установки. $a$ - генерация фотоэлектронов под действием вакуумного ультрафиолета, $b-$ характер поведения фотоэлектронов. Пояснения в тексте.

Письма в ЖТФ, 2018, том 44, вып. 18 
удаленной от фотокатода 1 на расстояние $L_{E G}$. Источником электронов является фотокатод, облучаемый вакуумным ультрафиолетом (VUV) с длиной волны $185-225 \mathrm{~nm}$. В оснастке 3 имеются окна 4 , в которых крепятся исследуемые образцы графена 5. Поток VUV поступает от внешнего источника, использующего дейтериевую лампу ДДС-30. Проходя через сеточный анод и графеновые мембраны, поток падает на поверхность фотокатода и генерирует в месте падения фотоэлектроны. Фотоэлектроны в вакууме $(2-5) \cdot 10^{-7}$ Torr под действием электрического поля, создаваемого источниками напряжения $V_{E}$ и $V_{G}$ (подключенными соответственно к фотокатоду и графеновой оснастке), двигаются в сторону графеновых мембран (рис. $1, b)$. Если бы фотоэлектроны не имели компоненты скорости $v_{g}$, параллельной мембранам, то они бомбардировали бы только мембраны. Однако $v_{g}$ в основном зависит от энергии кванта VUV и работы выхода электрона из алмаза. Максимальное значение этой скорости $v_{g \text { max }}$ оценивается следующим образом. Берется подложка с диаметром отверстия $D_{0}$. Проходя через отверстие, VUV-излучение рождает на фотокатоде в круге диаметром $D_{0}$ фотоэлектроны, которые под действием поля и благодаря отличной от нуля компоненте скорости $v_{g}$ образуют расходящийся поток, сечение которого у фотокатода имеет диаметр $D_{0}$, а у подложки $-D_{0}+2 \Delta L_{E G}$ (рис. $1, b$ ). Считая, что плотность электронов меняется линейно вдоль отрезка $\Delta L_{E G}$ и постоянна внутри отверстия, можно легко найти $v_{g \max }$ на основе измерения токов $I_{E}$ и $I_{G}$ при энергии электронов $50 \mathrm{eV}$. Величина $\Delta L_{E G}$ зависит от энергии электронов и при $L_{E G}=1 \mathrm{~mm}$ несложные вычисления дают $\Delta L_{E G}[\mu \mathrm{m}]=14\left(50 / E_{e}[\mathrm{eV}]\right)^{0.5}$.

Исследуемые образцы графена 5 представляют собой подложку со сквозными отверстиями, покрытыми однослойной или многослойной графеновой пленкой, что превращает ее в ряд мембран. Подложка крепится в окне оснастки 4 диаметром $D=2.4 \mathrm{~mm}$. В исследуемых образцах расстояние между мембранами менее $5 \mu \mathrm{m}$, а размытие электронного потока $\Delta L_{E G}$ происходит более чем на $14 \mu \mathrm{m}$, что усредняет падающий на подложку электронный поток, т. е. на поверхности подложки его можно считать равномерным, а вне подложки убывающим на расстоянии $\Delta L_{E G}$ по линейному закону. Отсюда легко вычисляется вероятность $p_{G}$ попадания фотоэлектрона на мембрану, a именно $p_{G}=\left(1+0.08 / E^{0.5}\right) S_{G} / S_{0}$, где $S_{G}$ - общая площадь мембран, $S_{0}=\pi D^{2} / 4$. Обозначая через $p_{T}\left(E_{e}\right)$ вероятность прохождения

7 Письма в ЖТФ, 2018, том 44, вып. 18 
мембраны электроном с энергией $E_{e}$, получаем, что вероятность $p_{e}$ для фотоэлектрона с энергией $E_{e}$ попасть на мембрану и пройти сквозь нее на анод будет $p_{e}=p_{T}\left(E_{e}\right) p_{G}$. Фотоэлектроны с вероятностью $\sim 1-p_{T}\left(E_{e}\right)$ также отражаются от мембраны. Таким образом, поток фотоэлектронов $J_{G E 2}$, падающий на мембраны, распадается на анодную составляющую $p_{T} J_{G E 2}$ и поток $\left(1-p_{T}\right) J_{G E 2}$ отраженных электронов. Часть падающих фотоэлектронов поглощается графеном, но, как показывает моделирование прохождения электронов через графеновую мембрану с помощью программы „Casino v2.48“, электроны в основном либо проходят, либо отражаются. Поэтому в рамках настоящей работы поглощение не учитывается. При средней величине угла отражения $\pi / 3$ получаем, что под действием тормозящего поля отраженный электрон попадает либо на подложку, либо на оснастку на расстоянии от места отражения $L_{R}=1.7 L_{E G}$. Таким образом, диаметр области возврата становится $D+3.4 L_{E G}$. Рассуждая точно так же, как в случае начального потока фотоэлектронов, получаем, что из всех отраженных электронов их часть $\eta_{R}$ снова попадает на мембраны, причем $\eta_{R}=0.33 S_{G} / S_{0}$. Часть отраженных электронов, попадая на мембрану, проходит через нее, а часть снова отражается. Вторым отражением можно пренебречь из-за значительного поглощения отраженных электронов подложкой и оснасткой. Электроны, вторично падающие на графеновую мембрану, падают под произвольными углами, а это, как упоминалось ранее, резко меняет всю картину поведения электронов из-за резонансных явлений $[9,10]$. Чтобы избежать усложнений, полагается, что вероятность прохождения фотоэлектронов не зависит от угла и равна $p_{T}\left(E_{e}\right)$. Тогда доля проходящих через мембрану первично отраженных электронов составит $\eta_{R} p_{T}\left(E_{e}\right)$. Итак, результирующий поток фотоэлектронов $I_{A f}$, проходящий через мембрану и достигающий анода, будет равен $I_{A f}=p_{e}\left(1+\eta_{R}-\eta_{R} p_{T}\right) I_{E f}$. При энергии электрона $E_{e}=e\left(V_{G}-V_{E}\right)$ ток $I_{E f}$ равен приращению тока $I_{E}$ при включении вакуумного ультрафиолета, а ток $I_{A f}$ равен разности $I_{A f}=I_{E f}-\Delta I_{G}$, где $\Delta I_{G}$ - приращение тока $I_{G}$ при размыкании-замыкании ключа 6 и непрерывном облучении VUV (рис. 1). Определяя коэффициент $K_{T R}\left(E_{e}\right)=I_{A f} / I_{E f}$, для нахождения $p_{T}$ получаем уравнение

$$
\left(1-0.08 / E_{e}^{0.5}\right) p_{T}\left(1+\eta_{R}-\eta_{R} p_{T}\right) S_{G} / S_{0}=K_{T R}\left(E_{e}\right)-K_{0},
$$

где $K_{0}$ - не зависящее от энергии электрона (при малых $E_{e}$ ) значение коэффициента $K_{T R}\left(E_{e}\right)$, обусловленное наличием незакрытых графеном 


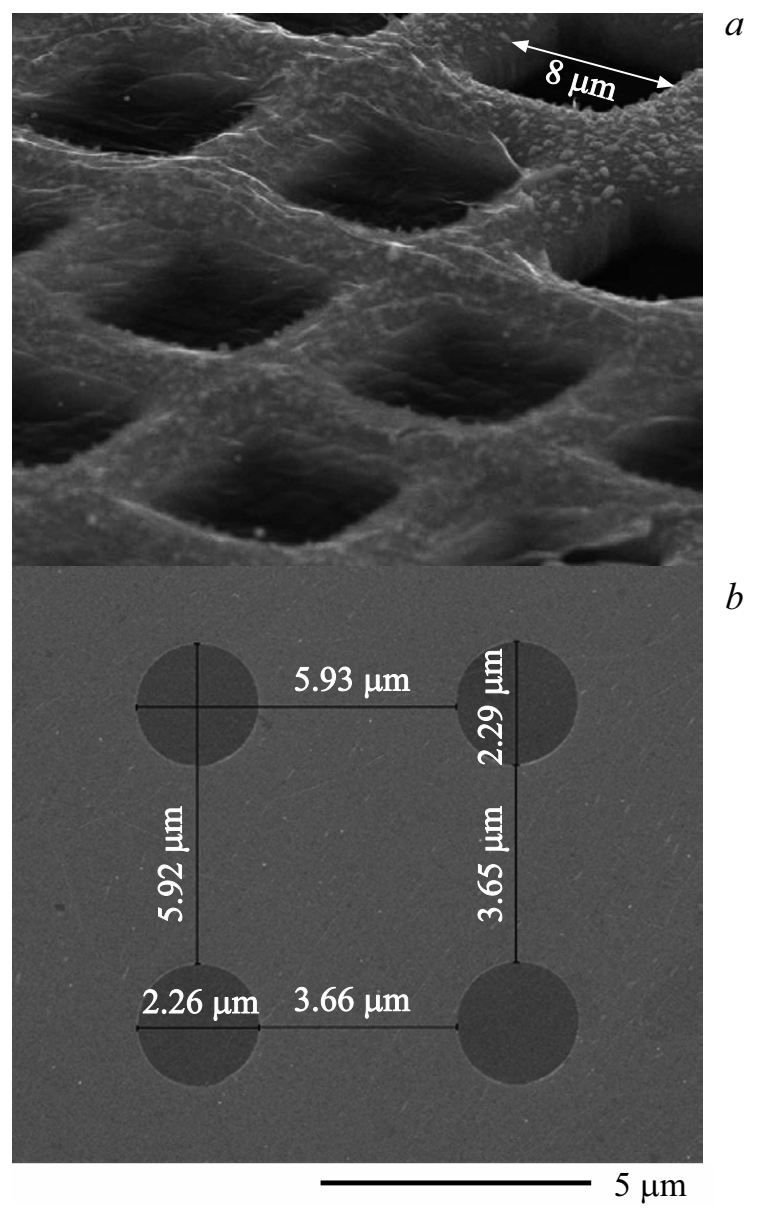

Рис. 2. СЭМ-изображения экспериментальных образцов. $a-$ Graphene Lab., $b-$ Graphenea Inc.

отверстий в подложке. Исследовались два образца, полученные от производителей Graphene Lab. и Graphenea Inc. CЭМ-изображение (СЭМ - сканирующая электронная микроскопия) первого образца представлено на рис. 2, $a$. Многослойная графеновая пленка (произво-

7* Письма в ЖТФ, 2018, том 44, вып. 18 

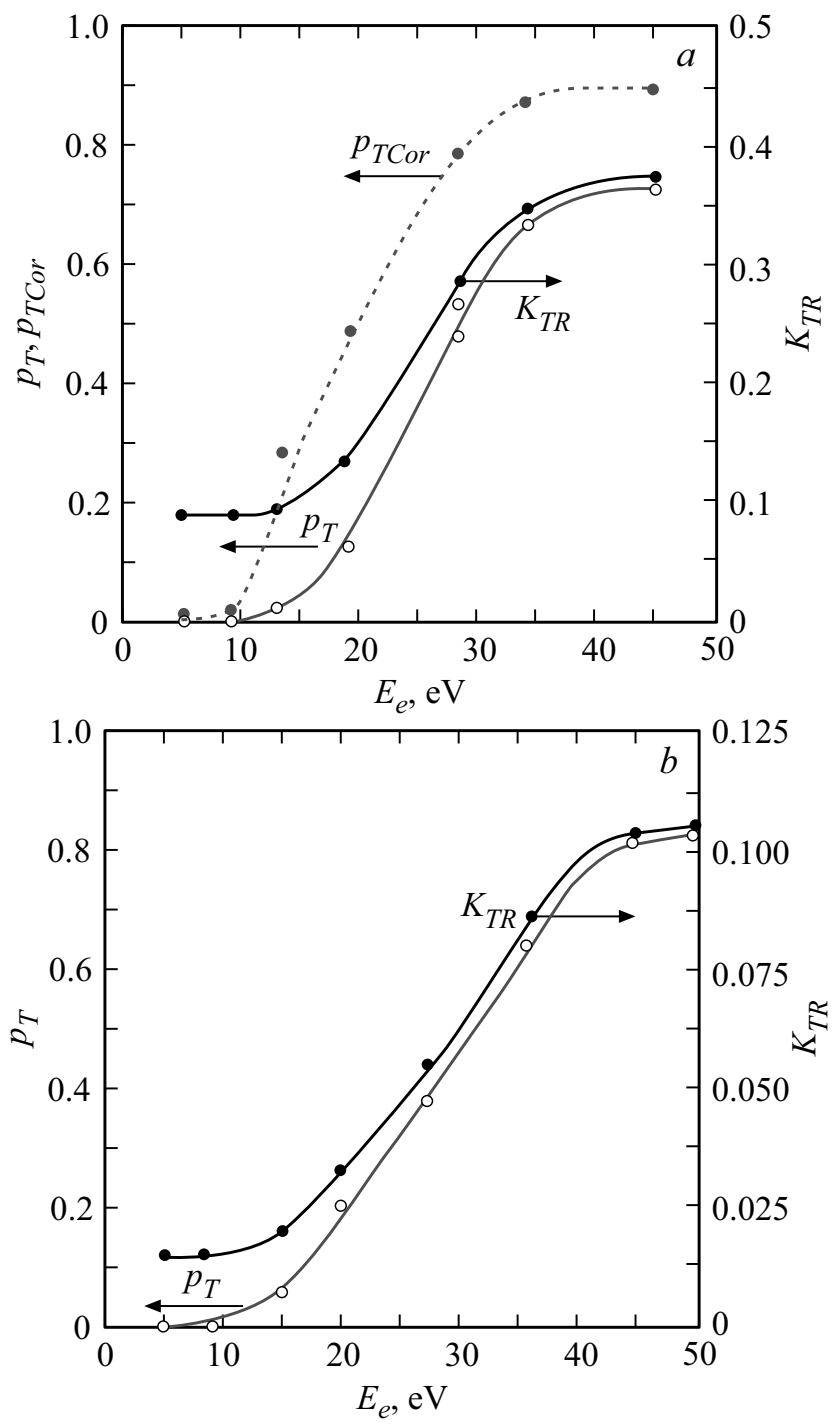

Рис. 3. Коэффициент прозрачности $K_{T R}$ и вероятности $p_{T}, p_{T C o r}$ прохождения электронов через несколько слоев и один слой графена для образца Graphene Lab. (a), через один слой графена для образца Graphenea Inc. $(b)$. 
дитель Graphene Lab. гарантирует, что число слоев графена по образцу может меняться только в пределах 1-6) нанесена на медную решетку с размером ячейки $8 \times 8 \mu \mathrm{m}$ и периодом $13 \mu \mathrm{m}$, отсюда $S_{G} / S_{0}=0.379$ и $\eta_{R}=0.12$. На рис. $3, a$ в виде кривой представлена полученная экспериментально зависимость $K_{T R}\left(E_{e}\right)$, причем $K_{0}=0.09$. Вероятность $p_{T}\left(E_{e}\right)$, вычисленная на основе уравнения (1), также представлена на рис. $3, a$; $p_{T}\left(E_{e}\right)$ - вероятность, отнесенная к нескольким графеновым слоям. Учитывая, что некоторые ячейки не покрыты графеном, а максимальное число слоев может достигать 6 , получаем, что среднее число слоев, приходящихся на одну ячейку, равно 3 . Исходя из средней величины слоев оцениваем вероятность $p_{T \text { Cor }}$ для одного слоя как $p_{T C o r}=p_{T}^{1 / 3}$ (рис. 3,a). Второй образец (рис. 2,b) представляет собой графитовую подложку с покрытыми однослойным графеном отверстиями диаметром $\sim 2.3 \mu \mathrm{m}$. Отверстия регулярно расположены с периодом $\sim 6 \mu \mathrm{m}$. Графитовая подложка совмещена с золотой решеткой, имеющей период $82 \mu$ и и ячейки размером $\sim 50 \times 50 \mu \mathrm{m}$. Образец имеет $S_{G} / S_{0}=0.119$ и с учетом золотой сетки $\eta_{R}=0.03$. Результаты измерений представлены в виде кривой $K_{T R}\left(E_{e}\right)$ на рис. $3, b$, причем $K_{0}=0.013$. Используя уравнение (1), получаем приведенную на рис. $3, b$ зависимость $p_{T}\left(E_{e}\right)$.

Отметим, что для двух образцов получены близкие результаты. Ocновным результатом является то, что при энергии электрона $40-50 \mathrm{eV}$ один слой графена становится практически прозрачным для электронов. Значение прозрачности при этих энергиях все же меньше единицы, что, по-видимому, обусловлено несовершенством графена и наличием на его поверхности „сопутствующих“ инородных частиц. При энергиях электрона 10-20 eV прозрачность графена падает существенно, но здесь остается открытым вопрос о том, преобладает поглощение или отражение. Использование для моделирования программы „Casino v2.48“ показывает превалирование процесса отражения. В нашем эксперименте явно преобладает процесс поглощения, что связано не столько со свойствами графена, сколько с подложкой.

Великолепные упругие свойства графеновых мембран и возможность их использования для стимуляции полевой эмиссии электронов позволяют уверенно прогнозировать применение графеновых пленок для разработки генерирующих и усилительных наноустройств СВЧ-диапазона вплоть до терагерцевого.

Работа выполнена при финансовой поддержке Министерства образования и науки РФ (проект № 8.3164.2017/ПЧ).

Письма в ЖТФ, 2018, том 44, вып. 18 


\section{Список литературы}

[1] Chen C., Lee S., Deshpande V., Lee G.-H., Lekas M., Shepard K., Hone J. // Nature Nanotechnol. 2013. V. 8. P. 923-927.

[2] Li C., Cole M.T., Lei W., Qu K., Ying K., Zhang Y., Robertson A.R., Warner J.H., Ding S., Zhang X., Wang B., Milne W.I. // Adv. Funct. Mater. 2014. V. 24. N 9. P. 1218-1227.

[3] Ильичев Э.А., Кулешов А.Е., Набиев Р.М., Петрухин Г.Н., Рычков Г.С., Сахаров О.А., Чернявская Е.С. // Письма в ЖТФ. 2013. Т. 39. В. 18. C. 25-31.

[4] Poltoratsky E.A., Rychkov G.S. // Nanotechnology. 2001. V. 12. P. 556-561.

[5] Hassink G., Wanke R., Rastegar J.T., Braun W., Stephanos C., Herlinger P., Smet J.H., Mannhart J. // APL Mater. 2015. V. 3. P. 076106.

[6] Mutus J.Y., Livadaru L., Robinson J.T., Urban R., Salomons M.H., Cloutier M., Wolkow R.A. // New J. Phys. 2011. V. 13. P. 063011.

[7] Frank L., Mikmekova E., Müllerova I., Lejenne M. // Appl. Phys. Lett. 2015. V. 106. P. 013117.

[8] Yan J.-A., Driscoll J.A., Wyatt B.K., Varga K., Pantelides S.T. // Phys. Rev. B. 2011. V. 84. P. 224117.

[9] Nazaron V.U., Krasovskii E.E., Silkin V.M. // Phys. Rev. B. 2013. V. 87. P. 041405.

[10] Wicki F., Longchamp J.-N., Latychevskaia T., Escher C., Fink H.-W. // Phys. Rev. B. 2016. V. 94. P. 075424.

[11] Longchamp J.-N., Latychevskaia T., Escher C., Fink H.-W. // Appl. Phys. Lett. 2012. V. 101. P. 113117. 